\title{
Investigation on the dispersal characteristics of liquid breakup in vacuum
}

\author{
X.X. Lu, ${ }^{1,2, a)}$ L. Li, ${ }^{1,4}$ K.H. Luo, ${ }^{3}$ X.B. Ren, ${ }^{1}$ Y. Liu, ${ }^{1,2}$ X.F. Yan ${ }^{1,2}$ \\ ${ }^{1}$ Beijing Pharmaceutical Chemistry Institute, Changping District, Beijing 102205, P.R.China \\ ${ }^{2}$ State Key Laboratory of NBC Protection for Civilian, Beijing 102205, China \\ ${ }^{3}$ Department of Mechanical Engineering, University College London, London WC1E 7JE, United Kingdom \\ ${ }^{4}$ Aerospace School, Tsinghua University, Beijing 100084, China
}

This work presents an experimental and numerical study on the dispersal characteristics of a liquid jet ejecting into vacuum. The liquid breaking is mainly caused by flash evaporation and bubbles expansion. Experiments of liquid dispersing under different conditions show that the superheat degree is the most important parameter influencing the pattern of the breaking liquid. The jet velocity has large influence on the distribution of the particle sizes. Mixing some volatile liquid with nonvolatile liquid can enhance the dispersion of the latter. The Lattice Boltzmann Method is applied to simulate the breakup process of liquid diglycol and the simulations have good agreement with the experimental results.

\section{INTRODUCTION}

Under certain conditions we encounter the problem of liquid dispersion in vacuum such as dumping waste liquid from the spaceship or space station into space, spray and phase change in a vacuum chamber of phase-change material, and so on. The mechanism of liquid breakup in vacuum is remarkably different from that in atmosphere. For a liquid like water, when the ambient pressure is lower than its saturated pressure, it becomes a superheated liquid. Superheat is an unstable or metastable state and the liquid trends to transfer into a more stable state, that is, gas or solid state. In this case, flash evaporation occurs. A number of experimental [1-15] and theoretical $[16,17]$ studies have been carried out on the behaviors of the flashing jet such as its stability or flow pattern, vaporization rate, temperature distribution, etc.

It is doubtless that the temperature of the liquid and the ambient pressure are the most important factors affecting the stability of the superheated jet. Experiments taken by Mann and Stoll [3], Funchs and Legge [4], etc., showed that the higher the temperature of water when leaving the nozzle is, the larger the spray angle is. Experiments taken by Kurschat, et al. [5], showed that the lower the ambient pressure is, the less stable the jet is. The initial temperature and the ambient pressure can form a parameter named Superheat Degree. There are two customary definitions for superheat degree of a liquid. One is defined as $\mathrm{H}$ which equals the ratio of the vapor pressure corresponding to the initial temperature of the liquid to the initial ambient pressure $(\mathrm{Ps}(\mathrm{T} 0) / \mathrm{Pe})[5]$. It is a dimensionless parameter. The other is defined as $\mathrm{S}$ which equals the difference of the initial temperature of the liquid and the saturation temperature corresponding to the initial ambient pressure $(\mathrm{T} 0-\mathrm{Ts}(\mathrm{Pe}))$ [6-8] and it has a dimension of $\mathrm{K}$. It can be seen that when Pe is given, both $\mathrm{H}$ and $\mathrm{S}$ increase with T0. On the other hand, 
when T0 is given, both $\mathrm{H}$ and $\mathrm{S}$ decrease with Pe. When neither Pe nor T0 is in the same variation between two cases, $\mathrm{H}$ and S may have different varying trends. Most of the existent experiments are carried out on the conditions that either initial temperature or the ambient pressure is fixed. Few have considered the cases of the same superheat degree but not temperature or pressure on its own.

Besides the superheat degree, the nozzle's shape and size also have been demonstrated to play an important role in the liquid breakup, showing that the stability of the jet decreases as the nozzle enlarges [9]. Mutair and Ikegami, 2009, 2010[7, 8] investigated effects of parameters including velocity of flow, initial temperature, superheat degree and injection nozzle diameter on the intensity of flash evaporation of the water jet. In their studies, spray from large tube (whose diameter is larger than $50 \mathrm{~mm}$ ) of low velocity (smaller than $3.6 \mathrm{~m} / \mathrm{s}$ ) and low superheat degree (where $\mathrm{S}<13 \mathrm{~K}$ ) was investigated. Cleary, Bowen and Witlox carried out series of experiments on the two-phase droplet dispersion of flashing liquid jets for superheat from 0K to $17.5 \mathrm{~K}$ and nozzle diameter from $0.75 \mathrm{~mm}$ to $4 \mathrm{~mm}[10,11]$. The global Sauter mean diameters (SMD) varying from $50 \mu \mathrm{m}$ to $80 \mu \mathrm{m}$ were measured through a PDA system in their studies and a formulation for the SMD was proposed by El-Fiqi et al [12].

In many cases, a liquid of high superheat degree cannot transfer to vapor completely except for a so-called retrograde liquid which has very large molar specific heat. The typical temperature-entropy diagrams for retrograde and normal liquid are shown in Fig. 1. The retrograde liquid can undergo a complete adiabatic evaporation when it is exposed to vacuum but the normal liquid cannot because it cannot enter the gas phase from the liquid phase through an isentropic path [5, 13]. For the normal liquid, violent flash evaporation usually causes freezing. Mikararian and Anderson's experiments showed that liquid jet ejecting into vacuum might freeze on a target plane [2]. Experimental studies conducted by Satoh et al. [14] demonstrated several possible phenomena of evaporation and corresponding congealment of liquid droplets, including stable surface evaporation, bubbling to freezing, bubbling to bursting and flashing to abrupt evacuation, etc. However, few works have mentioned about the freezing particles generated from the jet with high superheat degree.

(a)

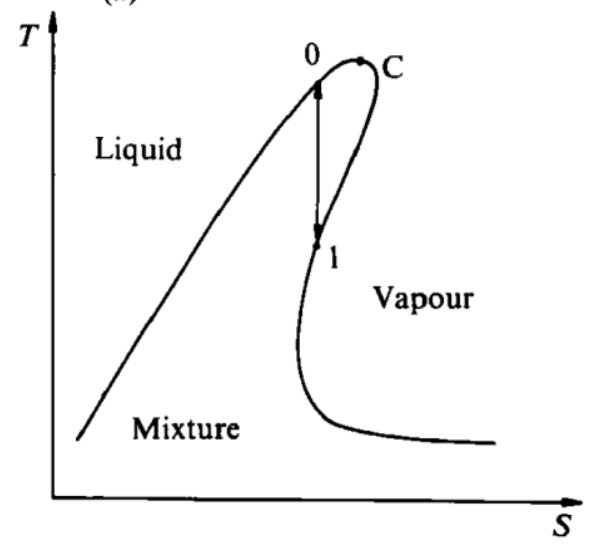

(b)

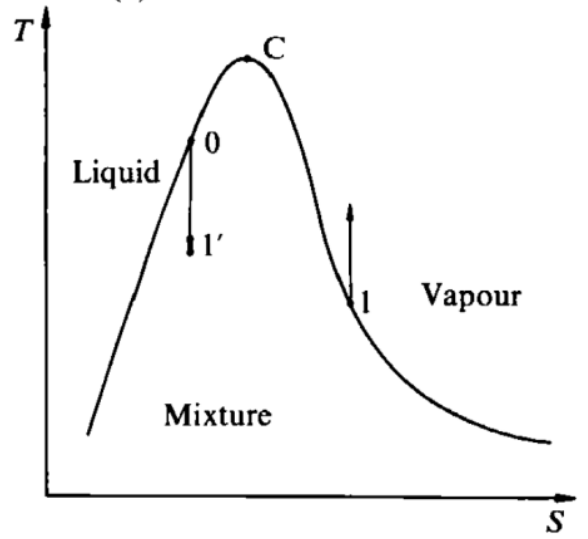

Fig. 1 Temperature-entropy diagrams for retrograde liquid (a) and normal liquid (b) [5]. 
Unlike the volatile liquid, the nonvolatile liquid jets may keep subcool and the breakup mechanism and flow pattern differ from those of flashing jet. For the absence of shearing with ambient air, the characteristics of nonvolatile liquid breakup in vacuum also differs from subcool jet in air. Gas bubbles expanding may play an important role in nonvolatile liquid breakup. Lior and Nishiyama carried out a research on the effect of gas bubbles on flash evaporation [18] but the influence of water fast evaporation cannot be excluded entirely in their experimental work. By the way, few works have mentioned the spray characteristics of the mixture of volatile and nonvolatile liquids and the generated droplets or particles.

There are also lots of numerical studies on the flash jet dispersion. Some of them focused on the thermodynamic state change of the liquid core using the continuum modeling method [19-23]. Others focused on the dispersion of two-phase flow using Lagrangian-Eulerian modeling method and solving the transport of discrete droplets or particles [24-26]. Few works focused on the process of liquid jet breakup in vacuum. One difficulty in numerical simulation of the fragmentation of liquid jet in vacuum is the complexity of the development of interfaces between different phases. From the bubbles growing and expanding, interacting with each other to liquid rupture, the interface topology changes rapidly and the scale is very small. The Lattice Boltzmann Method [27-29] (LBM) is a mesoscopic method for fluid flow simulation. The discrete Boltzmann equation is solved numerically instead of the Navier-Stokes equationa, so the LBM has a natural capability to deal with the interfaces and small scale flows because of its microcosmic character. The LBM has been applied to simulate two-phase flow successfully [30-33], but few have paid attention to the liquid breakup in vacuum.

In the present investigation, a flash chamber is designed to make an experimental research on the liquid jet breakup in vacuum. Several kinds of liquid under different pressure and temperature conditions are applied. In these experiments the influence of the superheat degree, spray velocity and the mass of the volatile liquid mixing in the nonvolatile liquid on the stability of the jet and the sizes of the droplets or the icing particles forming during liquid flashing dispersing are investigated. Applicability of the two definitions of superheat degree is discussed. Lastly the LBM is applied to simulate the fragmentation of a nonvolatile liquid jet in vacuum where bubbles expanding and bursting dominate the jet breakup.

\section{EXPERIMENTAL SETUP}

The experimental setup used for our study of liquid breakup in vacuum is shown as Fig. 2. A 160L tank with two visualization window is applied as the flashing spray chamber. The tank is cylindrical and made of stainless steel. The visualization windows are planar and made of quartz glass and they are convenient for illumination and image recordings. The dynamic pressure gauges are used to detect the pressure change in the chamber during the test. Because of the finitude of the capacity of the vacuum pump and the volume of the vacuum chamber, the ambient pressure recovers shortly after the liquid flash evaporation. So for the volatile liquid spray we could not investigate the behaviors of the jet in a constant low 
pressure circumstance, but under an initial condition of low ambient pressure. It can be seen that they have big influence on the pattern of the liquid jet during a short-time spraying (typically 10-30ms).

Distilled water, glycerol, diglycol and their mixture are chosen for the flashing spraying tests. For water spraying experiments, the bubble nuclei are removed from it through boiling up the liquid in the pretreatment process to exclude the effect of insoluble gas's expansion on the water jet breakup. In these experiments the initial pressure (P0) and temperature (T0) of the liquid and the initial ambient pressure (Pe) are variable parameters. The initial condition of the liquid is controlled by the pressure regulator and the temperature regulator. The ambient pressure in the chamber could be as low as $5 \mathrm{~Pa}$. The above-mentioned two definitions of the superheat degree will be discussed in detail.

The Redlake HG-100K high-speed camera is used to record the process of liquid break-up with the frame rate of 500fps or 1000fps. The core length and the sizes of the droplets or particles are measured by comparing with the outer diameter of the nozzle through post image processing.

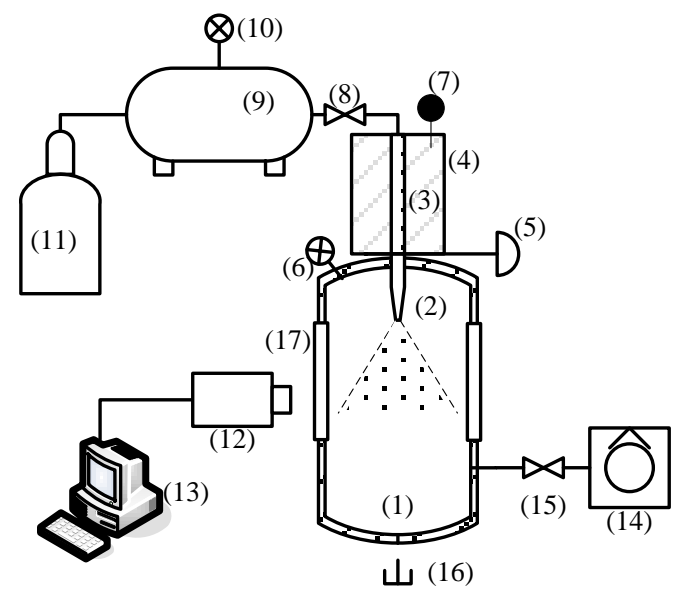

(1)Vacuum chamber; (2)Nozzle; (3)Liquid tube; (4)Temperature regulator; (5)Controlling valve; (6)Pressure gauge; (7)Thermometer; (8)Valve; (9)Pressure regulator; (10)Pressure gauge; (11)High pressure tank; (12)High speed camera; (13)Computer; (14)Vacuum pump; (15)Valve; (16)Drain valve; (17)Visualization window.

Fig. 2 Experimental setup

\section{EXPERIMENTAL RESULTS AND DISCUSSION}

\section{A. Characteristics of Water jets in vacuum with different superheat degrees}

The experimental conditions and measurements of the first series of tests are listed in Table 1 . The material is pure water and the diameter of the nozzle is $1.5 \mathrm{~mm}$. The only difference in the controlling conditions is the superheat degree. It can be seen that $\mathrm{H}$ of Case 1 in Table 1 is larger than that of Case 2 but $\mathrm{S}$ is on the contrary. 
Table I Main experimental conditions and results for water spraying

\begin{tabular}{cccccccc}
\hline \hline $\begin{array}{c}\text { Case } \\
\text { No. }\end{array}$ & $\begin{array}{c}\text { P0 } \\
(\mathrm{Bar})\end{array}$ & $\begin{array}{c}\mathrm{T} 0 \\
\left({ }^{\circ} \mathrm{C}\right)\end{array}$ & $\begin{array}{c}\mathrm{Pe} \\
(\mathrm{Pa})\end{array}$ & $\mathrm{H}$ & $\begin{array}{c}\mathrm{S} \\
(\mathrm{K})\end{array}$ & $\begin{array}{c}\text { Core length of jet } \\
(\mathrm{mm})\end{array}$ & $\begin{array}{c}\text { Visible } \\
\text { particles }\end{array}$ \\
\hline 1 & 1 & 2 & 5.4 & 129 & 50 & 5.4 & No \\
2 & 1 & 11 & 15 & 90 & 52 & 5.1 & No \\
3 & 1 & 30 & 15 & 280 & 71 & 2.5 & No \\
4 & 1 & 50 & 15 & 830 & 91 & 0 & Yes \\
\hline \hline
\end{tabular}

Figures 3-6 show the photographs taken by the high-speed camera with the frame rate of 1000fps for Case 1-4. Three photos in each case are given. Photos from left to right in each case correspond to $5 \mathrm{~ms}, 15 \mathrm{~ms}$ and $25 \mathrm{~ms}$ after the beginning time of ejection, respectively. It can be seen that the flow patterns for the first three cases become steady after a period of approximate $10 \mathrm{~ms}$ and the steady jets hold more than $15 \mathrm{~ms}$ before they are affected by recovery of the pressure in the chamber.

It could be seen that from case 1 to 4 the angle of the spray jet increases. The angle of the jet core region is about $46^{\circ}$ in case 1 and $160^{\circ}$ in case 4 . The core length of the jet decreases from case 1 to 4 on the contrary. These two parameters correlate to the stability of the jet. A smaller spray's angle and a longer core region of the jet represent a more stable jet. From the flow patterns in Fig. 3 and 4 it can be estimated that the jet in case 1 has a better stability than that in case 2. Generally speaking higher superheat degree should represent a less stable jet and more violent flash evaporation. So under this criterion $\mathrm{S}$ (the difference between temperatures) is seen to be more appropriate than $\mathrm{H}$ (the ratio of pressures) to characterize the behavior of the flashing jet.

The initial ambient pressure for all these 4 cases is lower than water's triple-point pressure (611Pa). Liquid water could not remain stable in such a low pressure circumstance and it should transfer into vapor or solid state completely. From water's temperature-entropy diagram it can be seen that for the adiabatic condition, normal substances like water cannot have all of it vaporized, but have a part of it solidified when it is exposed to such a low pressure environment. In other words the sensible heat of water could not supply enough latent heat needed for vaporization, so some of the liquid transforms into ice. Ice particles are detected in the chamber after experiments. It can be seen from Fig. 3-5 and the collected particles that the freezing particles generated from the first 3 cases are tiny, largest of which is smaller than $0.3 \mathrm{~mm}$.

The flow pattern for Case 4 differs from the first three cases greatly because of its high superheat degree. The flow just cannot remain a stable jet at all. The liquid breaks down as soon as it leaves the nozzle and the core region of the liquid jet is hardly seen. There are lots of visible freezing particles dispersing from the nozzle, some of which can be found in the chamber after experiments. The phenomenon of particles splashing lasts at least $50 \mathrm{~ms}$ in Case 4 and then the spraying jet resumes stable jet because of the recovery of the ambient pressure and decrease of the superheat degree. Sizes of these freezing particles are approximately of $0.8 \mathrm{~mm}$, a few even larger than $1 \mathrm{~mm}$. For high superheat degree, the liquid breaks up 
because of homogeneous nucleation and the rapid growth of the vapor bubbles. Both the nucleation rate and the vaporization rate are increment functions of superheat degree. Generally speaking, higher superheat degree corresponds to finer droplets generated from the flashing spraying, as indicted by the first three cases. In case 4 however, the superheat degree is high enough and vaporization in the liquid becomes so violent that the liquid drops freeze before they experience the secondary atomization and break up into finer droplets. As shown in Fig. 6, sizes of freezing particles generated in Case 4 are evidently larger than in cases $1-3$.
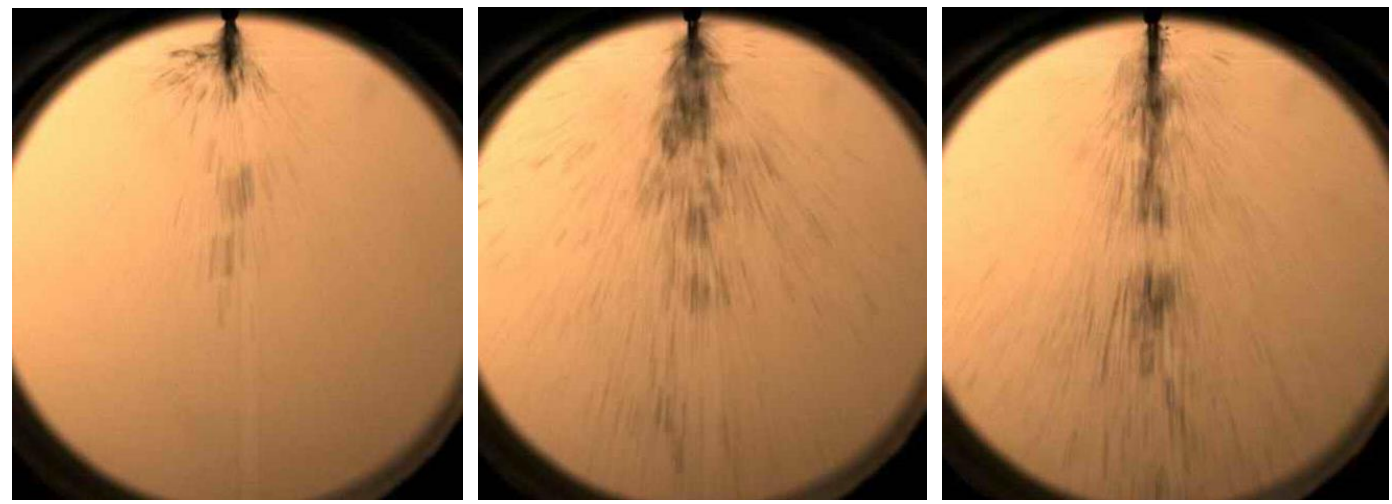

Fig. 3 Photographs taken from Case $1: \mathrm{Pe}=5.4 \mathrm{~Pa}, \mathrm{~T} 0=2^{\circ} \mathrm{C}$
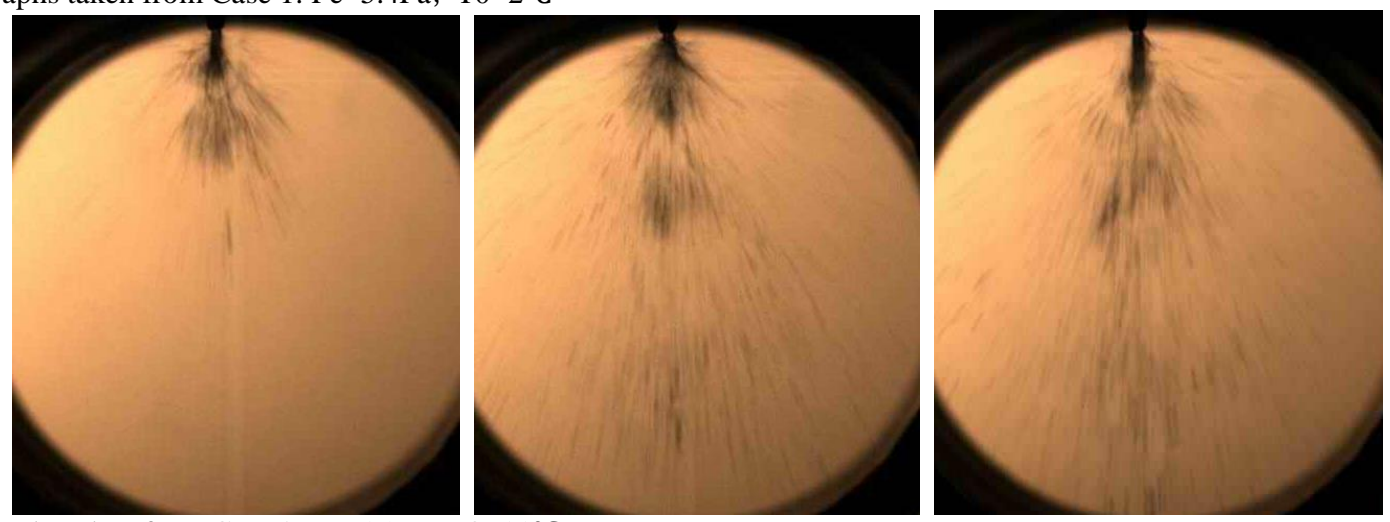

Fig. 4 Photographs taken from Case $2: \mathrm{Pe}=15 \mathrm{~Pa}, \mathrm{~T} 0=11^{\circ} \mathrm{C}$
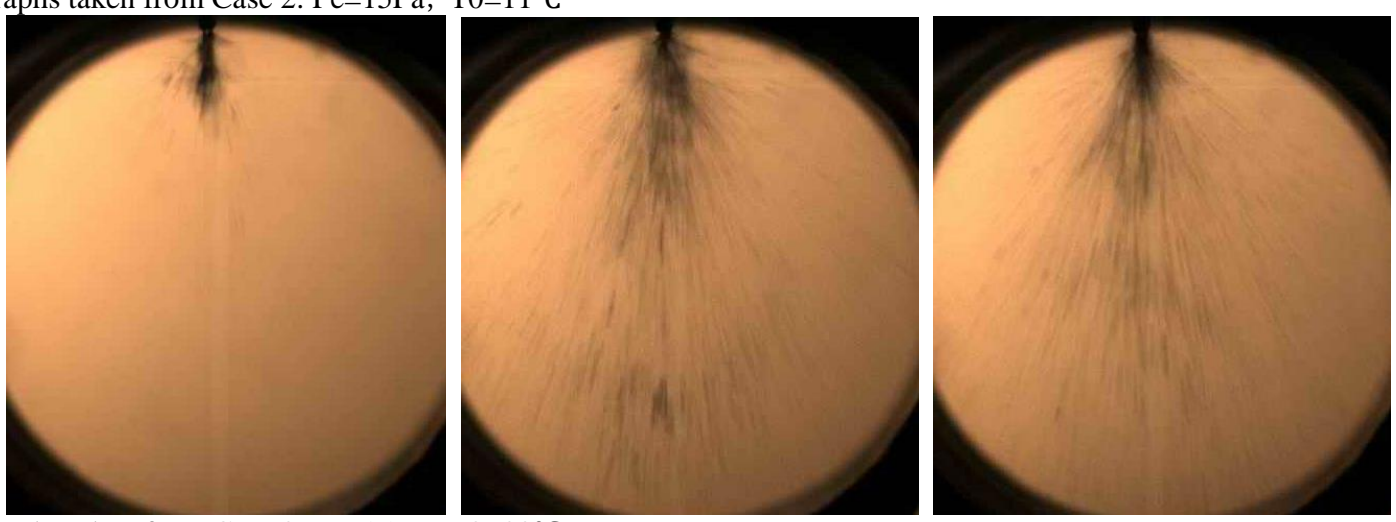

Fig. 5 Photographs taken from Case $3: \mathrm{Pe}=15 \mathrm{~Pa}, \mathrm{~T} 0=30^{\circ} \mathrm{C}$ 

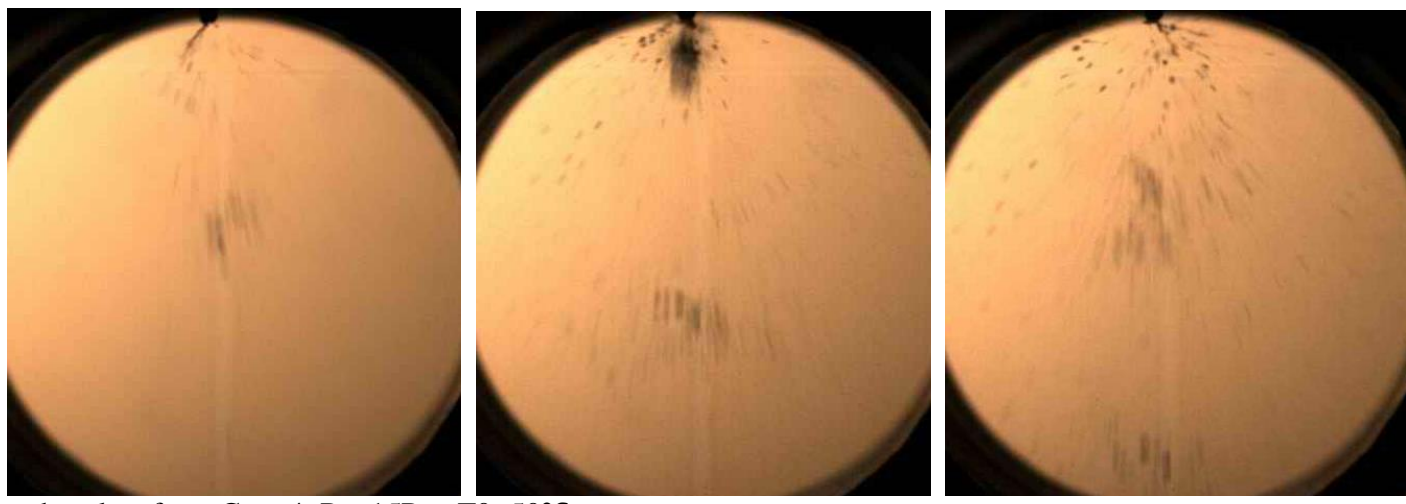

Fig. 6 Photographs taken from Case $4: \mathrm{Pe}=15 \mathrm{~Pa}, \mathrm{~T} 0=50^{\circ} \mathrm{C}$

\section{B. 3-2 Characteristics of diglycol jets in vacuum with different velocities}

Diglycol is a nonvolatile liquid with extremely low vapor pressure at normal temperature. Its saturated pressure at $120^{\circ} \mathrm{C}$ is only $1.389 \mathrm{kPa}$, in contrast to the saturated pressure of water at the same temperature of approximately $200 \mathrm{kPa}$. So the effects of jet's velocity on the flow pattern can be studied through this series of experiments.

The conditions of Cases 5 and 6 are shown in Table 2. The inner dimension of the nozzles used in these experiments is $4 \mathrm{~mm}$.

Table II Main experimental conditions and results for diglycol spraying

\begin{tabular}{ccccccc}
\hline \hline $\begin{array}{c}\text { Case } \\
\text { No. }\end{array}$ & $\begin{array}{c}\text { P0 } \\
(\mathrm{Bar})\end{array}$ & $\begin{array}{c}\text { T0 } \\
\left({ }^{\circ} \mathrm{C}\right)\end{array}$ & $\begin{array}{c}\text { Pe } \\
(\mathrm{Pa})\end{array}$ & $\begin{array}{c}\text { Core length of jet } \\
(\mathrm{mm})\end{array}$ & $\begin{array}{c}\text { Initial velocity } \\
(\mathrm{m} / \mathrm{s})\end{array}$ & $\begin{array}{c}\text { Half angle of jet } \\
\left({ }^{\circ}\right)\end{array}$ \\
\hline 5 & 1 & 15 & 40 & 5.4 & 7.0 & 0 \\
6 & 1.6 & 15 & 40 & 5.1 & 16.5 & 10 \\
\hline \hline
\end{tabular}

The flow pattern in Case 5 is shown in Fig. 7, the interval between adjacent photos is $6 \mathrm{~ms}$. The ambient pressure is kept at around 45Pa during the experiment and it can be seen that the total evaporation mass is negligible and so the diglycol jet is not a superheated jet. The half angle of jet at the nozzle is nearly $0^{\circ}$. The pattern of the jet does not change until the liquid diglycol is exhausted. The jet breaks down into some big droplets because of the distinct Rayleigh-Taylor (R-T) instability. The sizes of these droplets vary between $3 \mathrm{~mm}$ and $8 \mathrm{~mm}$, most of which are about $5 \mathrm{~mm}$. The residual bubbles in the liquid dilate and burst to make liquid membrane break up into much smaller droplets, the sizes of which are among $0.6 \mathrm{~mm}-1 \mathrm{~mm}$.

When the initial velocity increases to $16.5 \mathrm{~m} / \mathrm{s}$, the flow pattern of Case 6 is shown in Fig. 8 and remarkably different from that of Case 5. The liquid jet breaks down into lots of tiny droplets in this case. The maintenance of the ambient pressure under 45Pa suggests that the evaporation of the liquid can also be neglected and so the cause of the liquid breakup is not flashing but the velocity of the jet. There are two different patterns of the spray jet in this case. In the first $100 \mathrm{~ms}$ the half angle of jet at the nozzle is about $10^{\circ}$ and the liquid sprays like the flashing jet. According the estimate from the image processing, the sizes of the droplets generated in this period are below $0.5 \mathrm{~mm}$. The effect of bubble dilating and bursting on the liquid breaking is not distinctive and it can be presumed that the liquid breaks down due to the disturbances from the 
operation of the valve. After a period of about $100 \mathrm{~ms}$ the diglycol jet becomes more stable. The presence of the RayleighTaylor instability is discernible and some liquid ejects because of bubbles bursting.

Comparison between Case 5 and Case 6 shows the effect of jet's velocity on the nonvolatile liquid breakup in vacuum. When the jet is slow the bubbles' dilating and R-T instability dominate the liquid breakup and the sizes of the droplets are large. Otherwise when the velocity is high, the disturbances generated in the nozzle and valve lead the liquid break up drastically into tiny droplets.
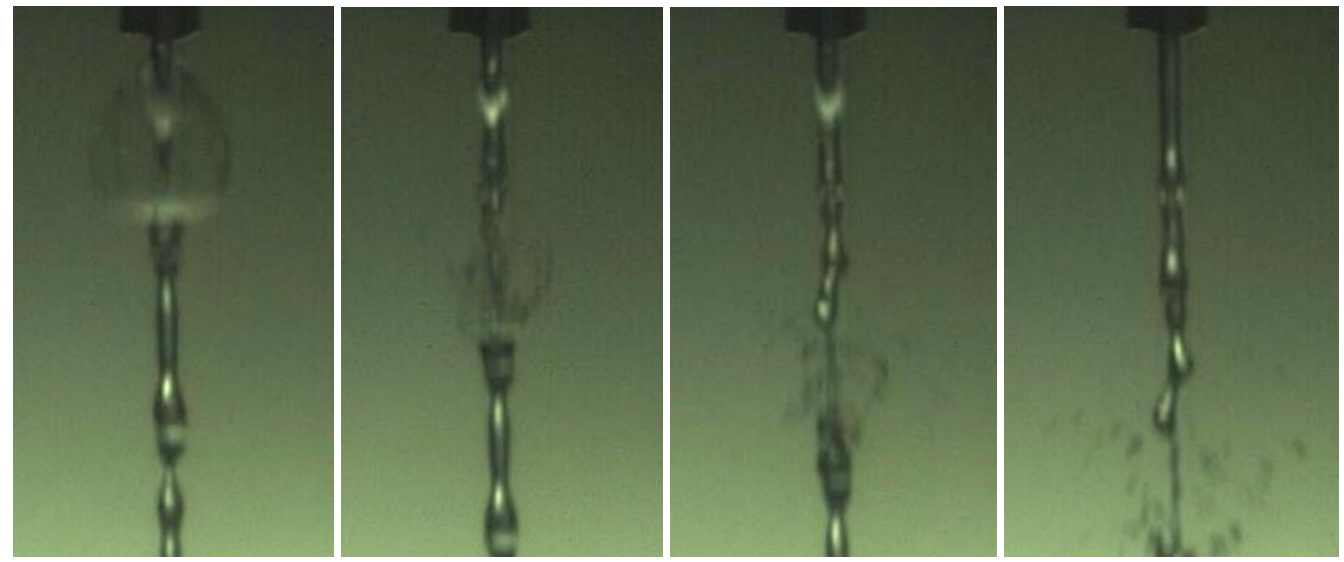

Fig. 7 Photographs taken from Case 5: Initial velocity $7 \mathrm{~m} / \mathrm{s}$
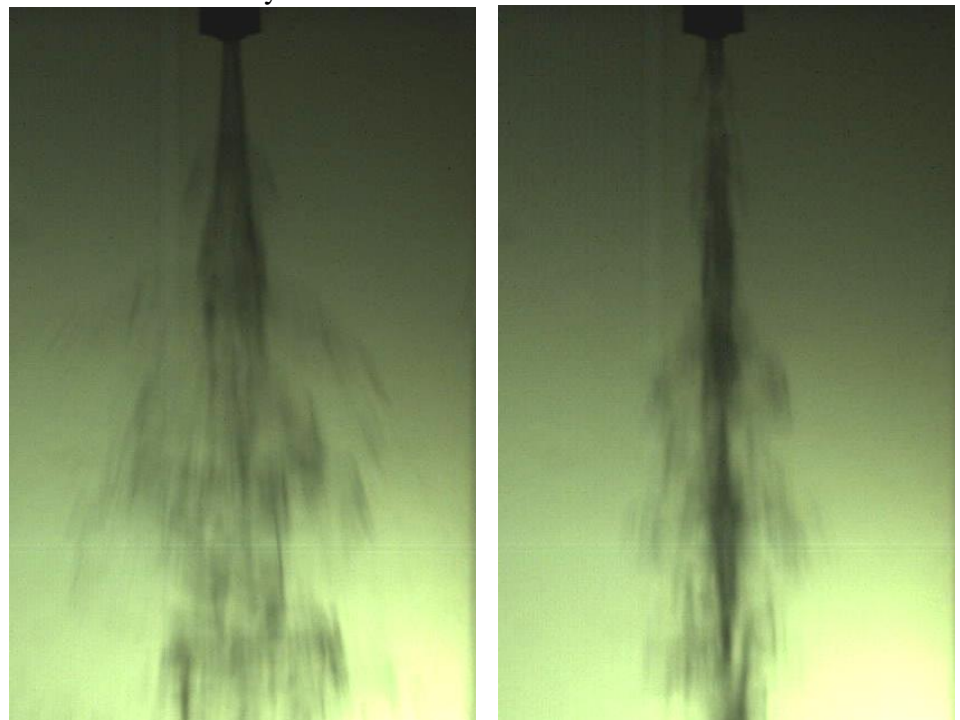

Fig. 8 Photographs taken from Case 6: Initial velocity $16.5 \mathrm{~m} / \mathrm{s}$. Left is the typical flow pattern in the former 100ms, right s the typical flow pattern after $100 \mathrm{~ms}$.

Comparison among experiments of diglycol and water spray in vacuum shows that fragmentation of liquid due to flash evaporation is far more violent than that due to disturbances and residual bubbles' dilating. In Case 6, the half angle of jet at the nozzle is only about $10^{\circ}$ when the half angle of the superheated jet in vacuum is much larger, some of the droplets ejecting even toward the rear of the nozzle. This is because liquid water must transform to gas or solid phase in such a low ambient pressure but liquid diglycol can exist.

\section{Characteristics of mixture of volatile and nonvolatile liquid jet in vacuum}


Experiments are carried out for spraying the mixture of volatile liquid and nonvolatile liquid. It can be the simulation for the spraying of nonvolatile liquid with various amount of mass of gas dissolved in it. When the liquid jet ejects into vacuum the volatile portion evaporates immediately and the vapor expands in the same way as the dissolved gas. Mixture of glycerol and water is applied here because one of them can dissolve in the other completely.

Table III Main experimental conditions for mixture of water and glycerol spraying

\begin{tabular}{cccccc}
\hline \hline $\begin{array}{c}\text { Case } \\
\text { No. }\end{array}$ & $\begin{array}{c}\text { Inner diameter of nozzle } \\
(\mathrm{mm})\end{array}$ & $\begin{array}{c}\mathrm{P} 0 \\
(\mathrm{Bar})\end{array}$ & $\begin{array}{c}\mathrm{T} 0 \\
\left({ }^{\circ} \mathrm{C}\right)\end{array}$ & $\begin{array}{c}\text { Pe } \\
(\mathrm{Pa})\end{array}$ & $\begin{array}{c}\text { Proportion between } \\
\text { glycerol and water }\end{array}$ \\
\hline 7 & 3 & 3 & 15 & 10 & 0 \\
8 & 1 & 4 & 15 & 10 & $3: 100$ \\
9 & 1.5 & 3 & 50 & 10 & $1: 3$ \\
\hline \hline
\end{tabular}

Fig. 9 shows the flow pattern of pure glycerol's jet in the vacuum (Case 7). The saturation pressure of glycerol is even lower than that of diglycol, so evaporation in this case can be neglected. Glycerol's viscosity is quite large, so the ejecting velocity is very low under the promoting pressure of 3Bar. The track of the glycerol jet is approximately due to the gravity, and the liquid column hardly expands or breaks up. A small quantity of residual gas nuclei grows and divides the liquid column into several segments.

Comparison between Case 5 and Case 7 shows the effect of liquid's viscosity on the breakup of jet. The amplitude of the instability wave on diglycol jet (Fig. 7) is evidently larger than that of glycerol's jet (Fig. 9), while the latter keeps a column shape within the tank's extent. This is because the glycerol's viscosity is much larger than that of diglycol. Viscosity plays an important role in restraining the growth of instability waves and the shearing and straining of the liquid. On the other hand, the inherence reason for liquid breakup is that there is shearing and straining existing inside the liquid. Therefore, it can be demonstrated that the viscosity plays a restraining role on nonvolatile liquid breakup in vacuum.

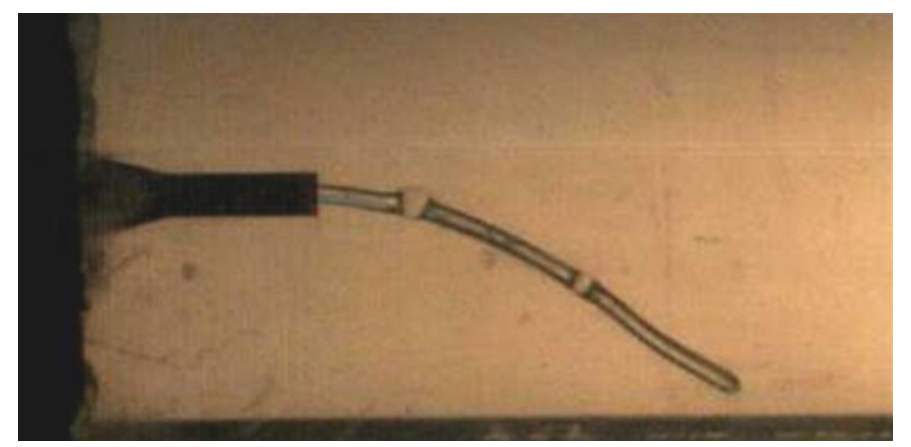

Fig. 9 Photographs taken from Case 7: Pure glycerol jet.

Figure 10 shows the flow pattern of the jet consisting of glycerol and a little water (Case 8). The proportion between water and glycerol is about 3:100. It can be seen that when the content of water in glycerol is very low, the glycerol jet could also maintain stability. Though water evaporates violently in vacuum, the jet's body would not break up entirely because of 
the low content of the volatile portion, but break up into some larger fragments. Callback of the droplets generated in this case shows that the sizes of glycerol droplets are about $1 \mathrm{~mm}$.

There cannot be freezing particles generating during dispersing of pure nonvolatile liquid in vacuum because there is hardly any evaporation which may absorb the sensible heat and cools the liquid. Adjusting the proportion of volatile liquid mixed in the nonvolatile liquid may make it possible that the nonvolatile liquid breaks up into droplets of suitable sizes and then freezes because of the flash evaporation of the volatile portion.
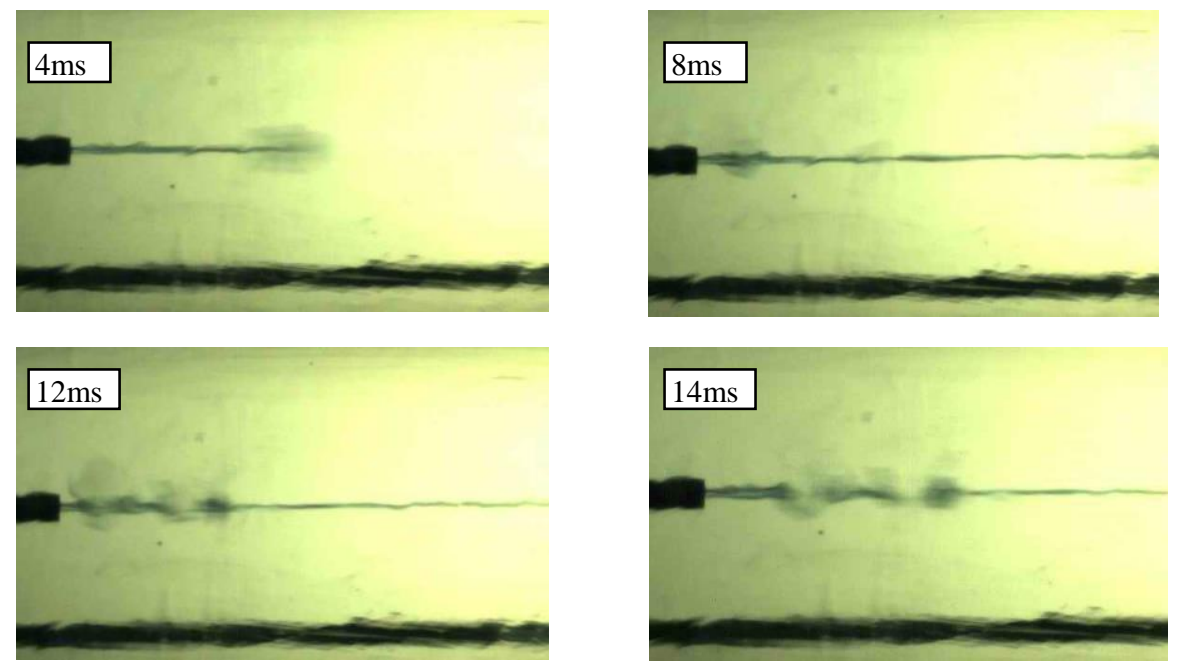

Fig. 10 Photographs taken from Case 8: Proportion between water and glycerol is about 3:100.

When the proportion of water is quite high (25\%), the flow pattern is like the superheated water jet, as shown in Fig. 11 (Case 9). It can be seen that flash evaporation of water plays a dominant role in the mixture jet's dispersing and the liquid breaks up into very tiny droplets.

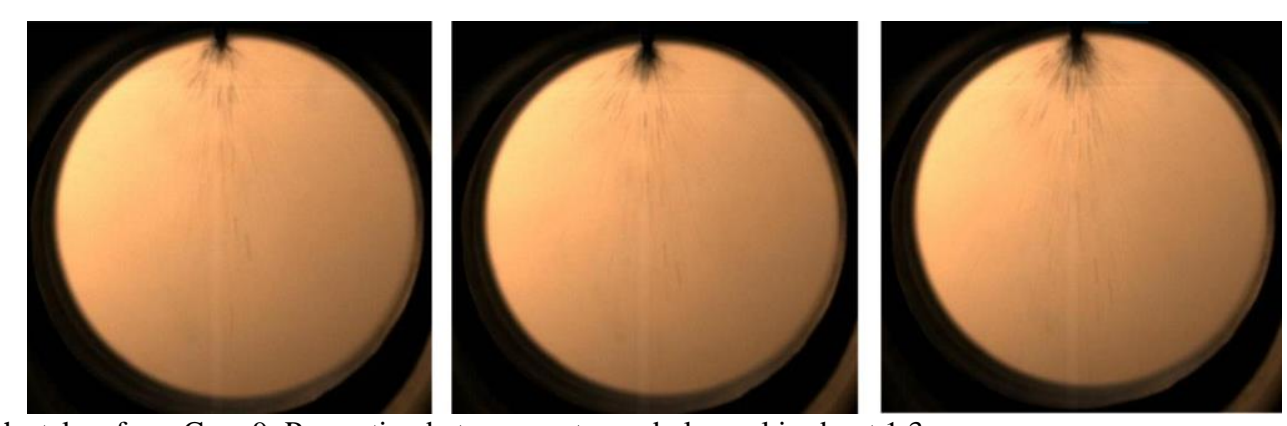

Fig. 11 Photographs taken from Case 9: Proportion between water and glycerol is about 1:3.

\section{VI.Numerical Simulation for Diglycol Breakup in Vacuum}

\section{A. Numerical model}

The Lattice Boltzmann Method (LBM) is applied here to simulate the breakup of diglycol jet in vacuum. The discrete Boltzmann equation including the BGK collisions model is as follows [34]: 


$$
f_{i}^{\sigma}\left(x+c_{i} \Delta t, t+\Delta t\right)-f_{i}^{\sigma}(x, t)=-\frac{1}{\omega^{\sigma}}\left(f_{i}^{\sigma}(x, t)-f_{i}^{\sigma(e q)}(x, t)\right)
$$

where $f_{i}^{\sigma}$ is the density distribution function for the i-th velocity and the $\sigma$ phase. $f_{i}^{e q}$ is the distribution function in equilibrium state which can be expressed by macro state values as follows in the assumption of homogenous temperature and low velocity.

$$
f_{i}^{\sigma(e q)}=w_{i} \cdot\left[1+\frac{e_{i} \times u}{c_{s}^{2}}-\frac{u^{2}}{2 c_{s}^{2}}+\frac{\left(e_{i} \times u\right)^{2}}{2 c_{s}^{4}}\right]
$$

In this case a rectangular two-dimensional nine-speed (D2Q9) lattice is applied and c denotes lattice velocity. Then $\rho^{\sigma}=\sum_{i} f_{i}^{\sigma}, \rho^{\sigma} u^{\sigma}=\sum_{i} c_{i} f_{i}^{\sigma}$. All the values are dimensionless. And the time step dt and lattice spacing $\mathrm{dx}$ can be decided through the following relation:

$$
\left(\tau-\frac{1}{2}\right) \times \frac{1}{3}=v \times \frac{d t}{d x^{2}}
$$

where $\tau$ is the relaxation time and $v$ is the macroscopic viscosity coefficient.

The Shan-Chen multiphase model is adopted in this work to model the interactions between different fluid phases $[35,36]$. The surface tension is expressed as follows:

$$
F(r)=-\tau G_{12} \psi(r) \sum_{i=1} w_{i} \psi\left(x+e_{i}\right) e_{i}
$$

where $\psi(r)=\rho_{0}\left(1-\exp \left[-\rho(r) \rho_{0}\right]\right), \mathrm{G}_{12}$ is the interparticle potential between phase 1 and phase 2 . The surface tension is then introduced into the LBM simulation by adjusting the macroscopic velocity in the collisions term as follows [37]:

$$
f_{i}(x, t+1)=f_{i}(x, t)+\frac{1}{\tau}\left\{f_{i}^{e q}[\rho(x, t), \quad u+F \tau]-f_{i}(x, t)\right\}
$$

A volume fraction $\varepsilon(x, t)$ is defined for every lattice for the model of interface reconstruction in two-phase flow. When $\quad \varepsilon\left(x+e_{i}, t\right)>\varepsilon(x, t) \quad, \quad d \varepsilon_{i}=\varepsilon\left(x+e_{i}, t\right)\left[f_{i}\left(x+e_{i}, t\right)-f_{i}(x, t)\right] \quad ; \quad$ otherwise, $d \varepsilon_{i}=\varepsilon(x, t)\left[f_{i}\left(x+e_{i}, t\right)-f_{i}(x, t)\right]$. We carry out the following operations to reconstruct the interfaces.

(1) If the volume fraction decreases to a negative value, assign the negative mass to the surrounding lattices according to the velocity distribution function to form a new boundary and let the volume fraction of the local lattice equal to 0 (empty lattice); 
(2) If the volume fraction exceeds 1, assign the excess part to the surrounding lattices according to the velocity distribution function and let the volume fraction of the local lattice equal to 1.

Let $f_{i}(x, t+1)=f_{i}^{e q}(u, \rho)$, that is to say, the fraction transported from the empty lattice to the interface lattice equals to the distribution function in the equilibrium state [38].

\section{B. Results and Discussion}

The processes of the growth and break-up of the bubble under different cases are investigated by simulation based on the above model. In the first case we assume that there is only one bubble generated in a single liquid drop. The diameters of the liquid drop and bubble are 30 and 18, respectively, which are corresponding to the real liquid drop of $3 \mathrm{~mm}$ and the bubble of $1.8 \mathrm{~mm}$ in diameter. The kinematic viscosity of the liquid is $1.03 \times 10^{-4} \mathrm{~N} / \mathrm{s}$, close to the value of diglycol. The process of the growth and breakup of the bubble in liquid droplet is shown in Fig.12. After the breakup of the bubble, tiny droplets of $0.1 \mathrm{~mm}$ are formed.
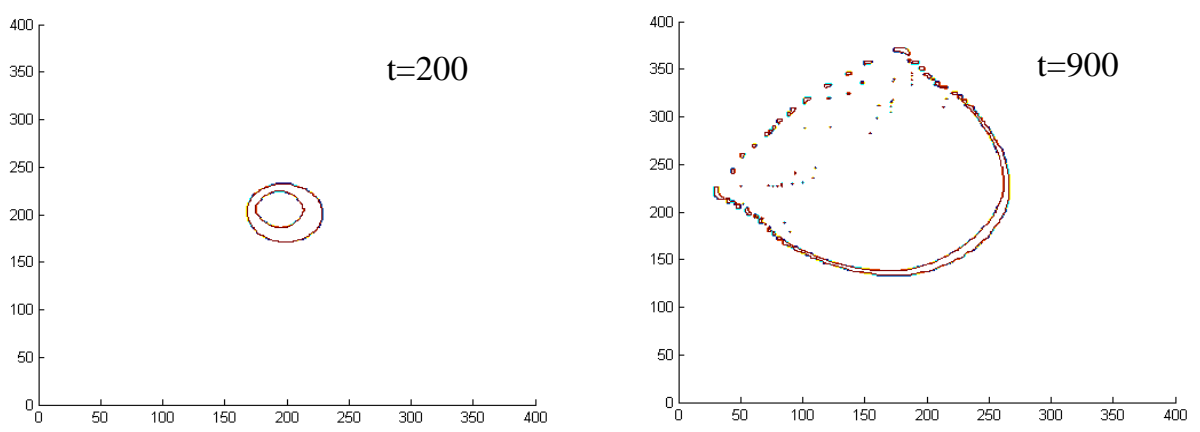

Fig.12 Single droplet with single bubble

In the second case, multiple bubbles are distributed randomly in the liquid jet. The process of the growth and breakup of the liquid jet caused by multiple bubbles bursting is indicated in Fig. 13. It can be seen that the results are consistent with diglycol ejecting experiment. The process is complex. At the outer boundary of the bubble, the formed droplets can be as small as $0.1 \mathrm{~mm}$ in diameter. On the other hand, droplets of larger diameter can be formed at the outer boundary of the two contacted bubbles because the expanding velocities of the bubbles decrease under the interaction of the flows.
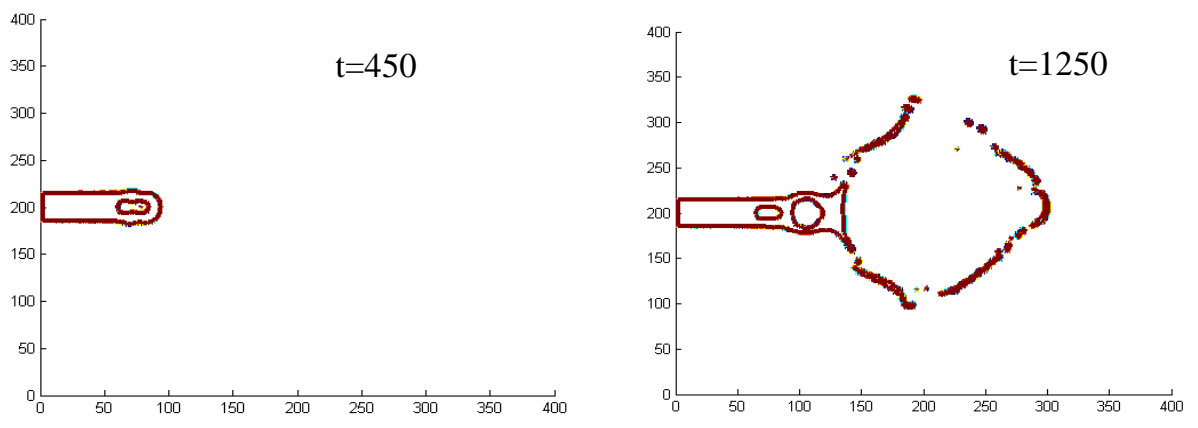

Fig. 13 Liquid jet breakup with multiple bubbles 


\section{Conclusions}

An experimental study on the dispersal characteristics of liquid jet ejecting in vacuum is carried out in this paper. The initial ambient pressure ranges between 5.4Pa and 40Pa. Water, diglycol and glycerol and their mixture were ejected into these low pressure environments. These experiments illuminate the effects of several parameters on the flow pattern and sizes of the generated droplets.

For volatile liquid such as water, the superheat degree plays an important role in the dispersal characteristics of the jet. For liquid jets of different initial temperatures but the same superheat degree, the flow exhibits similar patterns. For the two superheated degrees identified as the ratio of pressure and the difference between temperatures, the latter shows better capability in distinguishing the superheated flow's pattern and the characteristics of the generated freezing particles. When the superheated degree is high enough $(90 \mathrm{~K})$, the losing rate of the sensitive heat of the liquid due to violent flash evaporation is so fast that liquid freezes before it can break up into tiny droplets. For the nozzle of a size of $1.5 \mathrm{~mm}$, the sizes of generated freezing particles approach $1 \mathrm{~mm}$.

For nonvolatile liquid such as diglycol or glycerol, the main factors affecting the liquid breakup are the disturbances generated from the valve and the nozzle, and the growth of the residual gas nuclei. These factors also exist in the cases for volatile liquid, but in these cases they are comparatively less distinctive than the effects of flash evaporation. The LBM simulation reproduced the experimental observation and demonstrated the breakup process of the liquid and the formation of the droplets more clearly.

The jet of mixture of volatile and nonvolatile liquid can simulate the spraying of nonvolatile liquid with gas dissolved in it. Adjusting the proportion of volatile liquid mixed in the nonvolatile liquid may result in the breakup into droplets of suitable sizes and freezing of nonvolatile liquid's jet in vacuum possible.

\section{REFERENCES}

${ }^{1}$ R. Brown, J.K. York, "Sprays formed by flashing liquid jets," A.I.Ch.E. Journal 8, 149 (1962).

${ }^{2}$ R.R. Mikararian, R.G. Anderson, "An experimental investigation of a liquid jet expelled into vacuum," In: AIAA Unmanned Spacecraft Meeting 12, 255 (1965).

${ }^{3}$ B.L. Mann, O.T. Stoll, "Experimental investigation of the freezing problem presented in discharging water based fluids to space," In:Socienty of Automotive Engineers, 912 D, 1964.

${ }^{4}$ H. Funchs, H. Legge, "Flow of a water jet into vacuum," Acta. Astronautica 6, 1231 (1976).

${ }^{5}$ T.H. Kurschat, H. Chaves, G.E.A. Meier, "Complete adiabatic evaporation of highly superheated liquid jets," J. Fluid Mech 236, 43 (1992).

${ }^{6}$ J.H. Lienhard, J.B. Day, "The breakup of superheated liquid jets," Journal of Basic Engineering 9, 515 (1970).

${ }^{7}$ S. Mutair, Y. Ikegami, "Experimental study on flash evaporation from superheated water jets: Influencing factors and formulation of correlation," International Journal of Heat and Mass Transfer 52, 5643 (2009).

${ }^{8} \mathrm{~S}$. Mutair, Y. Ikegami, "Experimental investigation on the characteristics of flash evaporation from superheated water jets for desalination," Desalination 251, 103 (2010).

${ }^{9}$ E.P. Muntz, M. Dixon, "Application to space operations of free-flying , controlled streams of liquids," Journal of Spaceraft and Rockets 23, 411 (1986). 
${ }^{10}$ V. Cleary, P. Bowena, H. Witlox, "Flashing liquid jets and two-phase droplet dispersion I. Experiments for derivation of droplet atomisation correlations, " Journal of Hazardous Materials 142, 786 (2007).

${ }^{11}$ V. Cleary, P. Bowena, H. Witlox, "Flashing liquid jets and two-phase droplet dispersion II. Comparison and validation of droplet size and rainout formulations," Journal of Hazardous Materials 142, 797 (2007).

${ }^{12}$ A.K. El-Fiqi, N.H. Ali, H.T. El-Dessouky, H.S. Fath , M.A. El-Hefni, "Flash evaporation in a superheated water liquid jet," Desalination 206, 311 (2007).

${ }^{13}$ E. Hahne, G. Barthau, "Evaporation waves in flashing processes," International Journal of Multiphase Flow 26,531 (2000).

${ }^{14}$ I. Satoh, K. Fushinobu, Y. Hashimoto, "Freezing of a water droplet due to evaporation-heat transfer dominating the evaporation-freezing phenomena and the effect of boiling on freezing characteristics," International Journal of Refrigeration 25, 226 (2002).

${ }^{15}$ M. M. Vieira, J.R. Simões-Moreira, "Low-pressure flashing mechanisms in iso-octane liquid jets," J. Fluid Mech 572, 121 (2007).

${ }^{16}$ J.R. Simões-Moreira, M.M. Vieira, E. Angelo, "Highly Expanded Flashing Liquid Jets," Journal of Thermophysics and Heat Transfer 16, 415 (2002).

${ }^{17}$ G. Polanco, A.E. Holdø, G. Munday, "General review of flashing jet studies," Journal of Hazardous Materials 173,2 (2010).

${ }^{18}$ N. Lior, E. Nishiyama, "The effect of gas bubbles on flash evaporation," Desalination 45, 231 (1983).

${ }^{19}$ C.J. Knight, "Evaporation from a cylindrical surface into vacuum, " J. Fluid Mech 5, 469 (1976).

${ }^{20}$ E. Angelo, J.R. Simões-Moreira, "Numerical solution of highly expanded flashing liquid jets," Journal of Thermophysics and Heat Transfer 21, 379 (2007).

${ }^{21}$ H.T. Shin, Y.P. Lee, J. Jurng, "Spherical-shaped ice particle production by spraying water in a vacuum chamber," Applied Thermal Engineering 20, 439 (2000).

${ }^{22}$ Saurel R., Petitpas F., Abgrall R. Modeling phase transition in metastable liquids: application to cavitating and flashing flows[J]. J. Fluid Mech. 2008, 607:313-350.

${ }^{23}$ A. Zein , M. Hantke, G. Warnecke, "Modeling phase transition for compressible two-phase flows applied to metastable liquids, " Journal of Computational Physics 229, 2964 (2010).

${ }^{24}$ R.K. Calay, A.E. Holdo, "Modelling the dispersion of flashing jets using CFD," Journal of Hazardous Materials 154,1198 (2008).

${ }^{25}$ W. Tan, X. Liu, Y. Liu, Y. Bu, Z. Wang, "Modeling the two-phase cloud evolution from instantaneous flashing release using CFD," Journal of Loss Prevention in the Process Industries 24, 420 (2011).

${ }^{26}$ M. Ichard, O.R. Hansen, P. Middha, D. Willoughby, "CFD computations of liquid hydrogen releases, " International journal o f hydrogen energy 37, 17380 (2012).

${ }^{27}$ X. Shan, H. Chen, "Lattice BoItzmann model for simulating flows with multiple phases and components," Phys. Rev 47, 1815 (1993).

${ }^{28}$ M.R. Swift, W.R. Osborn, J.M.Yeomeans, "Lattice Boltzmann simulation of non-ideal fluids," Phys. Rev. Lett 75, 830 (1995).

${ }^{29}$ S. Chen, G. Doolen, "Lattice Boltzmann method for fluid flows," Annual Rev. Fluid Mech 30,329 (1998).

${ }^{30}$ D. Grunau, S. Chen, K. Eggert, "A lattice Boltzmann model for multiphase fluid flows," Phys. Fluids A 5, 2557 (1993).

${ }^{31}$ M.R. Swift, W.R. Osborn, J.M. Yeomans, "Lattice Boltzmann simulation of nonideal fluids," Phys. Rev. Lett. 75 , 830 (1995).

${ }^{32}$ X. He, S. Chen, R. Zhang, "A lattice Boltzmann Scheme for Incompressible Multi-phase Flow and Its Application in Simulation of Rayleigh-Taylor Instability," J. Comput. Phys. 152, 642 (1999).

${ }^{33}$ K.H. Luo, J. Xia and E. Monaco, "Multiscale modeling of multiphase flow with complex interactions," Journal of Multiscale Modelling, 1(2009) 125

${ }^{34}$ P.L. Bhatnagar, E.P. Gross, M .Krook, "A model for collision processes in gGases. I: small amplitude processes in charged and neutral one-component system," Phys. Rev. 94, 511 (1954).

${ }^{35}$ X. W. Shan and H. D. Chen, "Lattice Boltzmann model for simulating flows with multiple phases and components," Phys. Rev. E 47 (1993) 1815.

${ }^{36}$ X. W. Shan and H. D. Chen, "Simulation of nonideal gases and liquid-gas phase transitions by the lattice Boltzmann equation, " Phys. Rev. E 49 (1994) 2941.

${ }^{37}$ K. Sankaranarayanan, X. Shan, I.G. Kevrekidis, S. Sundaresan, "Bubble flow simulations with the lattice Boltzmann method," Chemical Engineering Science, 54(1999) 4817

${ }^{38}$ N. Thürey, T. Pohl, U. Rüde, M. Öchsner, C. Körner. "Optimization and stabilization of LBM free surface flow simulations using adaptive parameterization," Computers \&Fluids, 35(2006) 934 